\title{
COMPETITIVIDAD DE LAS MICRO Y PEQUEÑAS EMPRESAS (MYPES) ANTE EL TRATADO DE LIBRE COMERCIO (TLC)
}

\author{
COMPETITIVENESS OF MICRO AND SMALL COMPANIES IN THE PRESENCE OF \\ FREE TRADE AGREEMENT (NAFTA)
}

\author{
Elsa Esther Choy Zevallos* \\ Docente Asociado de la Facultad de Ciencias Contables, UNMSM
}

[Recepción: Enero de 2010 / Conformidad: Marzo de 2010]

\section{RESUMEN}

La participación de las Micro y Pequeñas Empresas (MYPES) Peruanas, ante la reciente apertura comercial, concretamente el Tratado de Libre Comercio (TLC), es casi nula, por tratarse de empresas que no son competitivas y que enfrentan muchos obstáculos para su desarrollo tales como: problemas de acceso a los mercados, barreras tecnológicas y dificultades para obtener recursos de crédito del sector financiero.

Frente a los desafíos de una Economía Abierta y Global, los Tratados de Libre Comercio (TLC) representan una oportunidad comercial para países como el Perú, ya que depende de otros países para alcanzar el crecimiento de su comercio exterior.

La apertura comercial y concretamente el Tratado de Libre Comercio, principalmente con Estados Unidos, es una oportunidad muy buena para las empresas peruanas que estén preparadas y una gran amenaza, para las que no lo estén; se requiere de competitividad, como así afirmó el ex director ejecutivo de USAID Mype Competitiva, Juan Carlos Mathews, al referirse al gran desafío de las Mypes peruanas frente al nuevo panorama económico.

Objetivo. El tema tiene por propósito señalar cuál es la situación de las Mypes y la posibilidad de participación ante el Tratado de Libre Comercio.

Material y método. Es un estudio descriptivo y de análisis de información relacionada

\begin{abstract}
The participation of Micro and Small Peruvians companies (MYPES), to the recent trade liberalization, particularly the Free Trade Agreement (NAFTA), is almost nil, because it is firms that are not competitive and they face many obstacles to its development such as problems of access to markets, technological barriers and difficulties in obtaining credit facilities from the financial sector. Faced with the challenges of an Open Economy and Global, the Free Trade Agreement (NAFTA) represent a commercial opportunity for countries like Peru, as it depends on other countries to achieve growth of foreign trade.

Trade liberalization and specifically the Free Trade Agreement, principally the United States, is a very good opportunity for Peruvian companies that are prepared and a great threat, for which they are not, requiring just competitiveness, as well said the former executive director of USAID MYPE Competitive, Juan Carlos Mathews, referring to the great challenge of the Peruvian Mypes front of the new economic landscape.

The issue has as objective to point out what is the status of MYPES and the possibility of participation to the free trade agreement. It is a study and analysis of information related to micro and small domestic firms in different economic sectors.

The competitiveness issue is of great interest in our country, especially as it relates to the
\end{abstract}

* Doctora en Ciencias Contables y Empresariales. Magíster en Contabilidad, UNMSM. Contadora Pública Colegiada Certificada. Docente Investigadora de la Facultad de Ciencias Contables, UNMSM. E-mail: eschoyz@yahoo.es 
con micro y pequeñas empresas nacionales de los distintos sectores económicos.

Conclusiones. El tema Competitividad es de gran interés en nuestro país, principalmente cuando se relaciona con las MYPES frente al Tratado de Libre Comercio, representando este un gran desafío para este tipo de empresas.

Palabras clave: Competitividad, micro empresas, pequeñas empresas, Tratado de Libre Comercio (TLC).

\section{LAS MYPES EN EL PERÚ}

En el Perú, las MYPES representan el 98.3\% del total de empresas existentes (94.4\% micro y $3.9 \%$ pequeña), pero el $74 \%$ de ellas opera en la informalidad. La gran mayoría de las MYPES informales se ubica fuera de Lima, generan empleo de mala calidad (trabajadores familiares en muchos casos no remunerados) con ingresos inferiores a los alcanzados en las empresas formales similares.

Las MYPES, además, aportan aproximadamente el $47.0 \%$ del PBI del país y son las mayores generadoras de empleo en la economía, aunque en la mayoría de los casos se trata de empleo informal.

En relación al desarrollo exportador del país, de las 6,656 empresas exportadoras, el $64.0 \%$ de ellas son MYPES, pero sobre el valor total exportado por el país, aproximadamente \$27,800 millones, las MYPES solo representan el $3.0 \%$.

Asimismo, hay responsabilidad del Estado peruano en relación a sobrecostos que claramente restan competitividad a la actividad empresarial en el país, por ejemplo, en Infraestructura (carreteras, puertos, entre otros) la desventaja es evidente, el flete aéreo, de Callao a Miami para un perecible fresco puede ser hasta $15 \%$ más costoso que un flete para el mismo producto de Santiago a Miami. Movilizar un contenedor (FCL) de 40'
MYPES against NAFTA, it represents a great challenge for these companies.

Key words: Competitiveness, micro enterprises, small businesses, the Free Trade Agreement (NAFTA)

en el puerto Callao cuesta por todo concepto, aproximadamente, $\$ 600$, mientras que en Valparaíso o Buenaventura puede costar hasta 30\% menos.

En Financiamiento, la MYPE peruana tiene limitaciones de acceso en la banca formal y de costo cuando puede acceder a una línea de financiamiento. Tasas de 35-40\% asumidas por muchas MYPES en el país hacen no competitivo su producto en el mercado internacional.

En relación a la Presión Tributaria, la alta carga tributaria es en parte el poco interés de muchas MYPES informales en el Perú de pasar a la formalidad. La MYPE es informal simplemente porque no entiende ni cree en las ventajas de formalizarse.

En relación a sus niveles de competitividad, existen serias deficiencias en cuanto a: Capacitación, costeo, negociaciones, formulación de planes de negocios, investigación de mercados, entre otros; estos factores representan algunos de los conceptos y herramientas de gestión poco manejadas por las MYPES peruanas y que son vitales para competir en mercados abiertos.

\section{ESTRATEGIAS MYPES COMPETITIVAS}

- Alianzas estratégicas. Algunas MYPES tienen la posibilidad de exportar en for- 
ma directa y pueden concretarse apelando a esquemas de asociatividad, como los consorcios. Algunas MYPES, probablemente, no exporten nunca, pero pueden participar en cadenas de exportación a través de subcontrata entre otras posibilidades de articulación empresarial. Las alianzas estratégicas significan una oportunidad para enfrentar la competencia.

- Soporte institucional. Existen muchos mecanismos de apoyo en gestión empresarial que ofrecen instituciones del estado como Promperú (ferias, inteligencia comercial), Produce (consorcios) y Mi Empresa del Ministerio de Trabajo y Promoción del Empleo (formalización). Existen también soporte de instituciones gremiales como ADEX, COMEX, Sociedad Nacional de Industrias, Cámara de Comercio de Lima, Perú Cámaras, etc.

Asimismo, existen varios programas de apoyo ofrecidos por organismos de cooperación empresarial que, directa o indirectamente, contribuyen o pretenden contribuir a la mejora de la competitividad de las empresas peruanas. USAID (Mype Competitiva y Proyecto PRA), Comisión Europea (Programa AL Invest), CAF (Programa Andino de Competitividad), CBI-Holanda, ICE-Italia, GTZ-Alemania, etc. En todos los casos, se trata de herramientas sub aprovechadas por las pequeñas empresas en el Perú.

- Marcas y patentes. Existe poco desarrollo de marcas y patentes por parte de las pequeñas empresas para generar valor agregado y sostenibilidad en sus negocios. Aún cuando, en los últimos años instituciones como The Global Entrepreneurship Monitor (GEM) consideró al Perú como el país con más índice de emprendimiento a nivel mundial (4 de cada 10 peruanos entre 18 y 65 años desarrolló actividades de emprendimiento, aunque en muchos casos por necesidad), significa un activo muy importante. Hay capacidad e idea para generar actividad empresarial.

\section{TRATADO DE LIBRE COMERCIO (TLC)}

Un tratado de libre comercio (TLC) es un acuerdo comercial vinculante que suscriben dos o más países para acordar la concesión de preferencias arancelarias mutuas y la reducción de barreras no arancelarias al comercio de bienes y servicios.

A fin de profundizar la integración económica de los países firmantes, un TLC incorpora además de los temas de acceso a nuevos mercados, otros aspectos normativos relacionados al comercio, tales como propiedad intelectual, inversiones, políticas de competencia, servicios financieros, telecomunicaciones, comercio electrónico, asuntos laborales, disposiciones medioambientales y mecanismos de defensa comercial y de solución de controversias.

Los TLC tienen un plazo indefinido, es decir, permanecen vigentes a lo largo del tiempo por lo que tienen carácter de perpetuidad.

\section{Tratado de Libre Comercio Perú}

Los Tratados de Libre Comercio forman parte de una estrategia comercial de largo plazo que busca consolidar mercados para los productos peruanos con el fin de desarrollar una oferta exportable competitiva que, a su vez, genere más y mejores empleos.

La experiencia muestra que los países que más han logrado desarrollarse en los 
últimos años son aquellos que se han incorporado exitosamente al comercio internacional, ampliando de esta manera el tamaño del mercado para sus empresas.

Por ejemplo, en los últimos años, los países del Asia (sin incluir Japón) incrementaron sus exportaciones a una tasa promedio anual de $6 \%$, lo que les ha hecho posible un crecimiento real de su economía de 5,5\% cada año. En contraste, en los últimos 30 años, América Latina (excluyendo México) ha presentado un bajo crecimiento real de sus exportaciones ( $1,9 \%$ anual) $y$, en consecuencia, un bajo crecimiento de su economía (2,9\% al año).

La necesidad de promover la integración comercial como mecanismo de ampliación de mercados es bastante clara en el caso del Perú, cuyos mercados locales, por su reducido tamaño, ofrecen escasas oportunidades de negocios y, por tanto, de creación de empleos.

\section{Objetivos del TLC PERÚ - EE.UU.}

- El TLC consolida el acceso preferencial del Perú a la economía más grande del mundo, lo que le permite ganar competitividad frente a otros países que no gozan de preferencias similares y ponerse en igualdad de condiciones frente a aquellos que sí gozan de ellas.

Un propósito en las negociaciones -que incluyen todos los aspectos de la relación económica bilateral- es obtener un acuerdo equilibrado e integral que beneficie el desarrollo de las cadenas productivas de la economía.

- Incrementary diversificar las exportaciones, eliminando distorsiones causadas por aranceles, cuotas de importación, subsidios y barreras para-arancelarias.
- Atraer flujos de inversión privada nacional y extranjera, propiciando el desarrollo de economías de escala, un mayor grado de especialización económica y una mayor eficiencia en la asignación de los factores productivos.

- Contribuir a mejorar la calidad de vida de las personas a través del acceso del consumidor a productos más baratos y de mayor calidad y variedad, la expansión de la oferta de empleo, el incremento de los salarios reales en el sector exportador.

- Establecer reglas claras y permanentes para el comercio de bienes y de servicios y para las inversiones, que fortalezcan la institucionalidad, la competitividad y las mejores prácticas empresariales en el país.

- Crear mecanismos para defender los intereses comerciales peruanos en Estados Unidos y definir mecanismos claros, transparentes y eficaces para resolver posibles conflictos de carácter comercial que puedan suscitarse.

- Reforzar la estabilidad de la política económica y de las instituciones, así como mejorar la clasificación de riesgo del Perú, lo que contribuirá a rebajar el costo del crédito y a consolidar la estabilidad del mercado de capitales.

- Reducir la vulnerabilidad de la economía a crisis financieras externas e incrementar la estabilidad de nuestros indicadores macroeconómicos, al estrechar vínculos con las tendencias de una de las economías más estables del mundo.

- Elevar la productividad de las empresas peruanas, al facilitarse la adquisición de tecnologías más modernas y a menores precios, que promueven la exportación de manufacturas y servicios con valor agregado. 


\section{EL TLC Y OPORTUNIDADES PARA LAS MYPES}

El Tratado de Libre Comercio (TLC) significa oportunidades para las micro y pequeñas empresas, la clave es saber segmentar el mercado, ya que las MYPE no pueden pretender entrar al mercado de grandes volúmenes de Estados Unidos. Su oportunidad es trabajar con productos de alto valor agregado. Es posible lograr eso con precios altos; hay posibilidades de venta en escala pequeña, siempre y cuando se maneje un alto estándar de calidad, e incluso una marca propia.

Se genera un conjunto de oportunidades que resulta interesante. Así, por ejemplo, se abre la oportunidad de que las MYPE importen maquinarias o equipos para la producción de los bienes y servicios que ofrecen a precios más bajos que los que han venido pagando hasta antes de la entrada en vigor del TLC. Por otro lado, también es importante considerar que así como el Perú reduce sus aranceles aplicables a la importación de bienes provenientes de ese país, Estados Unidos también hace lo propio permitiendo que los bienes producidos por las empresas peruanas, incluidas las MYPE, ingresen a ese enorme mercado cercano a los 300 millones de habitantes a precios mucho más competitivos.

Pero como se señaló anteriormente, si las MYPE alcanzan capacidad para hacer frente al reto que el TLC les plantea, entonces tomarán debida ventaja de las oportunidades que se les ofrece. Se espera que las MYPE, a las que muchos estudios les atribuyen la generación de más del 40\% del Producto Bruto Interno de nuestro país, tomen ventaja de esta oportunidad que se presenta con el TLC como consecuencia de abrirse para el Perú un mercado conformado no solamente por una población 10 veces mayor que la nuestra, sino que además se trata de una población con un ingreso per cápita casi 20 veces mayor que el nuestro.

\section{CONCLUSIONES}

1. Las mayores dificultades que enfrentan las PYMES de los países latinoamericanos es su baja capacidad administrativa para vincularse con el sector externo, la falta de información sobre oportunidades de exportaciones competitivas y sostenibles, con altos niveles de valor agregado local, como resultado de una mínima capacitación y gestión gerencial en el área internacional y su limitado acceso a tecnologías, especialmente las referentes al comercio exterior.

2. Los mayores obstáculos al proceso exportador de las MYPES en el Perú, se refieren a factores relacionados con el marketing, como son: la adecuación del producto a los requerimientos del mercado externo, la falta de información (conocimiento) de los mercados, el acceso a adecuados canales de distribución y la falta de capacitación en marketing internacional

3. El TLC abre, sin duda, oportunidades para las empresas peruanas. Depende de su capacidad para aprovecharlas; las nuevas generaciones en el Perú encaran un escenario muy distinto al que ha vivido cualquier peruano o residente en nuestro país en el pasado. De escenarios de inestabilidad política y económica, hiperinflación, terrorismo y, por ende, futuro incierto para cualquiera, hoy existe justificadas razones para sustentar una situación promisoria optimista en el Perú. 


\section{RECOMENDACIONES}

Es imperativo que el gobierno apoye a las Mypes a fin que estén preparadas para competir, convocando a los representantes para que conjuntamente se pueda analizar, evaluar, consensar proyectos de inversión, de ley, programas y propuestas viables y que conlleven al crecimiento y desarrollo empresarial.

\section{REFERENCIAS BIBLIOGRÁFICAS}

1. Eduardo Morón (2005). Evaluación del Impacto del TLC con Estados Unidos en la economía peruana. Centro de Inves- tigación de la Universidad del Pacífico. Marzo, Lima.

2. Eduardo Morón (2005). Tratado de Libre Comercio con los Estados Unidos: una oportunidad para crecer sostenidamente. Centro de Investigación de la Universidad del Pacífico. Diciembre, Lima.

3. Shank John K. y Govindarajan Vijay (1995). Gerencia estratégica de costos. Ed. Norma.

4. Convención Nacional del Agro Peruano. "Tratado de Libre Comercio entre Perú Estados Unidos”. Material de Trabajo.

5. Ministerio de Comercio Exterior y Turismo (2005). Preguntas y respuestas sobre el TLC Perú-Estados Unidos. Febrero, Lima. 